\title{
The significance of Computed Tomography in invasive paranasal mucormycosis*
}

\author{
Guy Slonimsky',\#, Einat Slonimsky 2,\#, Arkadi Yakirevitch', Doron Sagiv', \\ Shay Duvdevani', Yoav P. Talmi', Michael Wolf', Eran E. Alon' \\ ' Department of Otolaryngology-Head and Neck Surgery, the Chaim Sheba Medical Center, Tel Hashomer, Israel, \\ affiliated to the Sackler School of Medicine, Tel Aviv University, Israel \\ ${ }^{2}$ Department of Diagnostic Imaging, the Chaim Sheba Medical Center, Tel Hashomer, Israel, affiliated to the Sackler School of \\ Medicine, Tel Aviv University, Israe
}

Rhinology 56: 54-58, 2018

https://doi.org/10.4193/Rhino17.153

*Received for publication:

July 12, 2017

Accepted: September 10, 2017

\# Equal contribution

\begin{abstract}
Introduction: Early diagnosis of acute invasive fungal rhinosinusitis (AIFR) is crucial for patient's prognosis and may reduce the extent of surgical debridement. Initial evaluation usually includes paranasal Computed Tomography (CT), with an emphasis on bony erosion which is considered a specific but insensitive radiologic sign. Most studies made no distinction between Aspergillus and Mucor species while addressing CT findings. In this study, we seek to evaluate whether bony erosion on paranasal CT is a significant and reliable finding in the initial evaluation of invasive paranasal mucormycosis.
\end{abstract}

Methods: A retrospective review of pre-operative non-contrast craniofacial CT scans of patients diagnosed with acute invasive fungal rhinosinusitis (AIFR) caused by Mucor species for the presence of bony erosion.

Results: A total of 13 patients (9 males, 4 females) were included. Twelve patients were immunosuppressed due to various hematological malignancies. Six patients underwent debridement due to gross intraoperative findings of bony fungal invasion, but only one patient had evidence of bony erosion on the pre operative paranasal CT.

Conclusion: Bony erosion on paranasal $\mathrm{CT}$ is an exceptionally insensitive radiologic sign for establishing or rejecting the diagnosis of Mucor induced AIFR. The mainstay of confirming or rejecting the diagnosis of AIFR is by physical examination, endoscopy and oriented biopsy of suspicious mucosal lesions.

Key words: diagnostic techniques, respiratory system, paranasal sinus diseases, sinusitis

\section{Introduction}

Acute invasive fungal rhinosinusitis (AIFR) is an uncommon, rapidly progressive life threatening disease, almost exclusively afflicting immunocompromised hosts. Although multiple fungal species can cause acute invasive fungal disease, the most common isolated pathogens are of Aspergillus and Mucor species (1). Historically, reported mortality rates reached $50-80 \%{ }^{(2)}$, and despite improved imaging modalities, surgical techniques and medical treatments, mortality rates still approximate $50 \%(3,4)$. Nonetheless, in many patients with AIFR, mortality is associated with the underlying medical condition ${ }^{(1)}$.

Presenting signs and symptoms of AIFR, include fever, nasal congestion and discharge, mucosal necrosis, facial swelling, periorbital cellulitis, proptosis, facial hypoesthesia, headache, ophthalmoplegia, loss of visual acuity and deteriorated mental status in advanced disease ${ }^{(3,5,6)}$. Prompt diagnosis and aggressive treatment by the combination of surgical debridement, antifungal pharmacotherapy and if possible, termination of the immunocompromized state, are the keys to improved outcome and survival ${ }^{(7)}$.

A thorough inspection of the palatal mucosa, nasal endoscopy with biopsies of ischemic/necrotic lesions, and paranasal sinuses computed tomography (CT), are considered the mainstay of the initial evaluation in cases of suspected AIFR. Diagnosis is confirmed by histopathologic evidence and culture of invasive fungi. MRI was suggested as beneficial for the evaluation of intracra- 
nial involvement ${ }^{(6)}$.

Initial CT findings in patients with AIFR are usually nonspecific and may resemble other forms of acute or chronic rhinosinusitis which include unilateral mucosal thickening, air/fluid levels, mucoperiosteal thickening and infiltration of the periantral fat planes ${ }^{(8-11)}$. Bony erosion with extension of disease beyond the paranasal sinuses, although mainly seen in advanced cases, was suggested as a specific CT finding and even as a hallmark of AIFR $(8,10,12,13)$. Most studies made no distinction between Aspergillus and Mucor species while addressing CT findings.

The goal of the present study was to evaluate the role of bony erosion as a significant and reliable CT finding in cases of invasive mucormycosis with Mucor species. Our data underscores the fact that bony erosion is not a consistent dependable sign of Mucor induced AIFR and its absence does not exclude the possibility of severe life threatening disease.

\section{Materials and methods}

A retrospective review of craniofacial CT scans of patients diagnosed with AIFR caused by Mucor species between the years 2007-2016 at the Chaim Sheba Medical Center was performed. Patients with aspergillosis were excluded.

All patients had histopathologic diagnosis of AIFR with the identification of Mucor species either by histology and/or culture. CT scans were obtained prior to surgical debridement.

A single patient was diagnosed by endoscopy only due to poor general condition which demonstrated necrotic tissue and by fungal culture. Non contrast CT scans were obtained in the coronal and axial planes.

CT studies were initially evaluated by the attending head and neck neuroradiologist at the day performed and were retrospectively reviewed by a blinded senior radiologist (ES) with an emphasis on the presence of bony erosion.

All CT studies were performed on the following CT scanners: Mx8000 Quad 4-slices, Mx8000 IDT 16-slices, Brilliance 40, 64 and 128 (Philips Medical Systems, Eindhoven, the Netherlands), and 64-slice VCT LightSpeed (GE Medical Systems, Milwaukee, WI, USA).

All CT studies were obtained in axial planes with coronal reconstruction using bone and soft-tissue algorithms.

Images were acquired with 0.9-1.25 mm thick sections and spacing of $0.45-0.70 \mathrm{~mm}$ and in-plane field of view (FOV) of 250 $\mathrm{mm}$. Coronal reconstructions using bone algorithm were also performed at a section thickness of 0.90-1.25 mm with 0.63-1.00 $\mathrm{mm}$ spacing. In one patient, the CT study was acquired with 2.50 $\mathrm{mm}$ thickness and spacing of $0.63 \mathrm{~mm}$ with coronal reformations.

Bony erosion was defined as breaks in the cortical bone surface, and was evaluated as a dichotomy variable either present or absent.

Extent of disease was evaluated by the initial endoscopic and ra-
Table 1. Patient characteristics.

\begin{tabular}{|c|c|c|c|c|}
\hline Patient & Age (Years) & Sex & $\begin{array}{l}\text { Underlying } \\
\text { medical } \\
\text { condition }\end{array}$ & $\begin{array}{l}\text { Absolute neu- } \\
\text { trophil count } \\
\text { (cell/ } / \mu \mathrm{L} \text { ) }\end{array}$ \\
\hline 1 & 20 & $\mathrm{M}$ & $A M L^{*}$ & 2,790 \\
\hline 2 & 20 & $\mathrm{~F}$ & $\mathrm{HL}^{+}$ & 2,000 \\
\hline 3 & 10 & M & $\mathrm{ALL}^{\ddagger}$ & 800 \\
\hline 4 & 21 & $\mathrm{M}$ & ALL & 2,000 \\
\hline 5 & 9 & $\mathrm{M}$ & ALL & 20 \\
\hline 6 & 4 & $\mathrm{~F}$ & ALL & 300 \\
\hline 7 & 5 & $\mathrm{M}$ & ALL & 400 \\
\hline 8 & 17 & M & ALL & 600 \\
\hline 9 & 61 & $\mathrm{~F}$ & $\mathrm{CLL}^{\S}$ & 34,000 \\
\hline 10 & 63 & M & MDS" & 5,400 \\
\hline 11 & 49 & M & None & 15,000 \\
\hline 12 & 61 & $\mathrm{~F}$ & AML & 40 \\
\hline 13 & 67 & $M$ & $\mathrm{NHL}^{*}$ & 120 \\
\hline
\end{tabular}

AML*, acute myeloblastic leukemia; HL†, Hodgkin's lymphoma; ALL‡, acute lymphocytic leukemia; CLL§, chronic lymphocytic leukemia; MDSף, myelodisplastic syndrome; NHL\#, non Hodgkin's lymphoma

diological findings either on CT and/MRI, and staged according to a survival-correlated scale as suggested by Talmi et al. ${ }^{(4)}$ : Stage I: Disease localized to nose only with minimal tissue invasion.

Stage II: Disease limited to nose, ipsilateral sinuses, and orbit. Stage III: Disease involving intracranial structures with unimpaired or limited impairment of cognition.

Stage IV: Disease involving intracranial structures with unconsciousness or hemiplegia, bilateral disease, skin necrosis, palatal involvement.

\section{Results}

Thirteen patients (9 males, 4 females), were included, mean age was 31 years (range 4-67 years). Mean interval from paranasal CT to tissue sampling was 3.2 days. Demographics, underlying medical condition and absolute neutrophil counts are summarised in Table 1.

All patients had paranasal CT scans performed prior to endoscopy/tissue sampling. Twelve patients had consecutive imaging studies performed during follow-ups, either CT scans and/or MRI's.

Extent of disease (staging by Talmi et al. ${ }^{(4)}$ ), interval from CT to biopsy/surgical debridement and the presence of bony erosion revealed by CT scans are summarised in Table 2 . 
Table 2. Staging, interval from CT to tissue biopsy and the presence of bony erosion.

\begin{tabular}{|cccc|}
\hline Patient & Stage & $\begin{array}{c}\text { Interval to Biopsy } \\
\text { (days) }\end{array}$ & $\begin{array}{c}\text { Bone erosion on } \\
\text { CT* (Yes/No) }\end{array}$ \\
\hline 1 & II & 2 & No \\
\hline 2 & IV & 2 & No \\
\hline 3 & IV & 4 & No \\
\hline 4 & IV & 4 & No \\
\hline 5 & II & 0 & No \\
\hline 6 & IV & 2 & No \\
\hline 7 & IV & 6 & No \\
\hline 8 & I & 2 & Yes \\
\hline 9 & IV & 7 & No \\
\hline 10 & II & 3 & No \\
\hline 11 & IV & 9 & No \\
\hline 12 & I & 1 & No \\
\hline 13 & & 3 & \\
\hline
\end{tabular}

$C T^{*}$, computed tomography.

Twelve patients were immunocompromised due to underlying hematological malignancies, five of whom died of their underlying medical condition. Interestingly, one patient was a previously healthy 49 years old male (patient \#11) who worked as a carpenter and presented with headache, right facial swelling and numbness, without fever or nasal discharge. Physical examination demonstrated mild right facial edema, trismus and hypoesthesia over the distribution of the right V2. Paranasal CT scan demonstrated bilateral mucosal thickening of the maxillary and sphenoid sinuses, bilateral infiltration of the pterygoid muscles and infratemporal fossae with no bony erosion (Figure 1). Tissue biopsy demonstrated invasive mucormycosis. Subsequent MRI's demonstrated extensive bilateral infiltration of the skull base, infratemporal fossae, masticator spaces, pre-vertebral space and cavernous sinuses with intracranial extension. Due to a far advanced disease revealed by MRI, the patient was treated conservatively with antifungal medications with full recovery. Five patients $(\# 2-4,7,9)$ presented with invasive fungal involvement of the hard palate and underwent extensive bony debridement. All preoperative paranasal CT scans did not demonstrate palatal bony erosion (Figure 2).

A 9 year old male (patient \#5) with ALL and invasive mucormycosis involving the lateral wall of the left nasal cavity, had a prior invasive sinonasal aspergillosis with minimal involvement of the left middle turbinate, 5 months earlier. He then underwent partial resection of the left middle turbinate and treated with Vori-

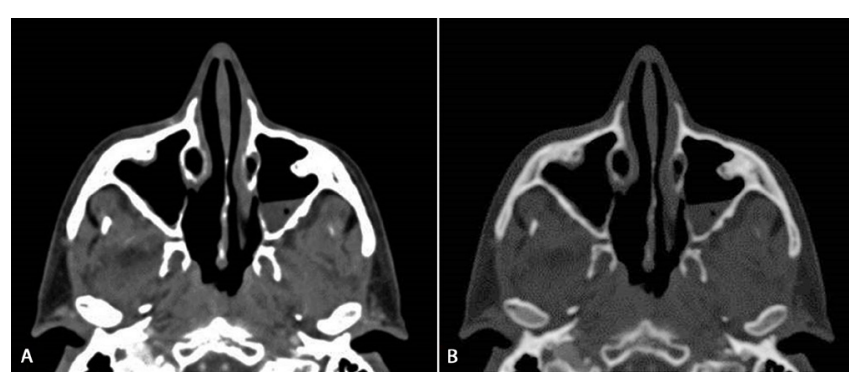

Figure 1. A) Axial contrast-enhanced paranasal CT scan displays bilateral mucosal thickening of the maxillary sinuses and infiltration of the pterygoid muscles and infratemporal fossae. B) Same axial plane in bone window, with no evidence of bony erosion.

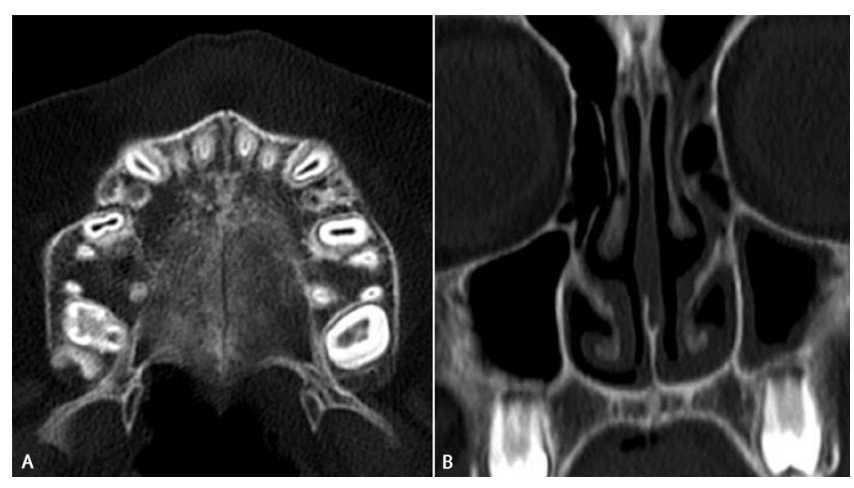

Figure 2. Paranasal non contrast CT scan of Patient \#3, a 10 year old male with ALL and intraoperative findings of left alveolar ridge, premaxilla and hard palate necrosis. The patient underwent infrastructure maxillectomy. A) Axial view of the hard palate and alveolar ridge with no evidence of bony erosion. B) Coronal view.

conazole. He presented with fever, pain over the left maxillary sinus and mild left periorbital cellulitis. The CT scan performed at the day of the endoscopic debridement demonstrated left pansinusitis with no bony erosion. Endoscopy revealed mucosal necrosis of the medial and inferior turbinates, medial wall of the left maxillary sinus, anterior ethmoids and frontal recess. Tissue biopsy and culture confirmed the diagnosis of invasive mucormycosis. The patient was treated with Amphotericin B and underwent 2 additional endoscopies under general anesthesia with no evidence of residual disease.

We found evidence of preoperative bony erosion on paranasal CT scan in only one case $(7.7 \%)$ of a 17 year old male (patient \#8) with T-cell acute lymphocytic leukemia (ALL). The patient presented with left periorbital cellulitis and painful induration of the left aspect of the nasal bridge. Paranasal CT scan performed 2 days pre op demonstrated erosion of the left nasal bone (Figure 3). Nasal endoscopy and debridement revealed necrotic mucosa over the anterior aspect of the left middle turbinate, and necrotic bone at the left pre lacrimal region underneath intact mucosa. The patient eventually recovered with a combination of Amphotericin B and Posaconazole, with no need for 


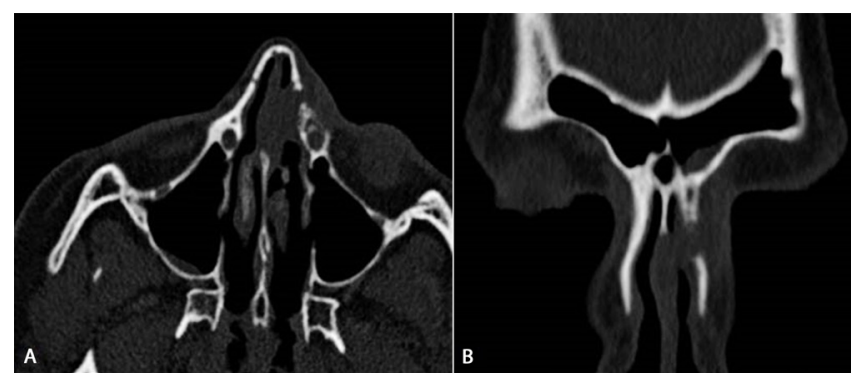

Figure 3. A, Axial non contrast paranasal CT scan displays erosion of the left nasal bone and lacrimal apparatus. B, Coronal view.

additional debridement.

Overall, Six patients underwent bony debridement due to fungal invasion identified in the operating room, but only one patient (17\%) had evidence of bony erosion on the pre operative paranasal CT.

\section{Discussion}

Diagnosis of AIFR in its earlier stage is of paramount importance in regard to the patient's prognosis and the extent of surgical debridement. At an early stage, limited sinonasal debridement with antifungal medications and successful termination of the immunocompromised state if possible, will most likely lead to resolution of the invasive disease. A delayed diagnosis may lead to increased potential for extensive craniofacial resection, or to a deteriorated patient with an inoperable fatal disease.

The initial evaluation of patients suspected for having AIFR includes a thorough head and neck examination with an emphasis on nasal endoscopy and tissue biopsy for pathology and microbiology studies. Unless intracranial, base of skull or orbital involvement is clinically suspected, MRI is not routinely obtained. In most of the cases, a paranasal CT scan was already performed prior to the consultation with the otolaryngologist. Nonspecific findings resembling acute or chronic rhinosinusitis are the prevalent findings ${ }^{(8-11)}$ and therefore, have no influence upon the diagnostic and or therapeutic algorithm. Finkelstein et al. ${ }^{12)}$ reviewed various paranasal CT findings of 14 patients with hematological malignancies and AIFR ( 6 with Aspergillus species and 8 with Mucor species). Radiologic findings were compared to a control group of 20 patients with suspected but finally excluded AIFR. The authors concluded that in early stages of AIFR, paranasal CT findings are nonspecific and can suggest or reject AIFR with a high probability predominantly in advanced stages. Groppo et al. ${ }^{(14)}$ compared MRI and CT imaging in 17 immunocompromised patients with AIFR and concluded that MRI has a relatively higher sensitivity in early stages of disease.

In a review of the literature regarding radiologic features of invasive and noninvasive fungal sinusitis, Aribandi et al. ${ }^{(9)}$ stated that in cases of invasive disease, bony erosion is a specific feature of an advanced infection and therefore may be subtle in many cases. The fungi tend to progress alongside vessels or nerves ${ }^{(15,}$ 16) and consequently has the capability to extend beyound the sinuses through intact bone ${ }^{(9)}$.

Middlebrooks et al. ${ }^{(13)}$, retrospectively reviewed CT findings of 42 patients diagnosed with AIFR, 42.9\% (18 patients) with Aspergillus species, and $23.8 \%$ (10 patients) with Mucor species. They reported $100 \%$ specificity and $35 \%$ sensitivity for bony erosion as a marker of disease. Interestingly, although bony erosion is considered an indicator of advanced disease, it was not evident in all 7 patients who eventually died of AIFR. Similar results were described by DelGaudio et al. ${ }^{\left({ }^{(10)}\right.}$ which reported CT findings of bony erosion in 8 out of 23 patients with AIFR, 14 with Aspergillus species, and 9 with Mucor species ${ }^{(10)}$. Both studies, did not specify the prevalence of bony erosion for Mucor species versus Aspergillus species individually.

Cho et al. ${ }^{(17)}$, reported significantly increased serum galactomannan levels in patients with Aspergillus AIFR compared to patients with Mucor AIFR and Aspergillus fungus ball. The authors suggested using galactomannan levels as a noninvasive tool for early diagnosis of patients with Aspergillus AIFR as well as for discrimination of fungal species which is of paramount importance for guiding therapy with the appropriate antifungal agent. We present 13 patients diagnosed with AIFR, exclusively with Mucor species. In 6 cases, necrotic bone was identified and debrided in the operating room. Our review of their paranasal CT scans revealed evidence of bony erosion merely in one case. Furthermore, the lack of evidence of bony erosion on paranasal CT scan in the presence of gross intraoperative findings of palatal involvement emphasizes its low sensitivity for bony erosion. We believe that the findings in our series, underscore the fact that bony erosion on paranasal CT is an exceptionally insensitive radiologic sign for establishing or rejecting the diagnosis of Mucor induced AIFR. Other previously described nonspecific findings can indicate laterality and progression of the invasive fungal infection. Seasonal fluctuation should also raise the index of suspicion ${ }^{(18)}$.

Despite that, paranasal CT is still playing a pivotal role in the diagnostic and therapeutic algorithm during initial evaluation, endoscopic surgical planning and follow up of patients with suspected AIFR, with MRI reserved mainly for cases with suspected intracranial, base of skull or orbital involvement.

In the context of the immune compromised patient, a high index of suspicion should be the rule, even in light of "benign" non-specific CT findings.

\section{Conclusion}

Based on our experience, we come to the conclusion that the mainstay of confirming or rejecting the diagnosis of AIFR is by physical examination, endoscopy and oriented biopsy of suspicious mucosal lesions. Bony erosion is not a consistent dependable sign of Mucor induced AIFR and its absence does not 
exclude the possibility of severe life threatening disease.

\section{Acknowledgements}

No financial support or funding was provided.

\section{Authorship contribution}

GS, ES: principle researchers, collection, analysis, and interpre- tation of data, writing of manuscript. AY, DS, SD, YPT, MW: study design, writing and review of manuscript. EEA: study design, writing, review, and approval of final draft.

\section{Conflict of interest}

Authors declare no conflict of interests.

\section{References}

1. Parikh SL, Venkatraman G, DelGaudio JM Invasive fungal sinusitis: a 15-year review from a single institution. Am J Rhinol. 2004 Mar-Apr;18(2):75-81.

2. Denning DW, Stevens DA. Antifungal and surgical treatment of invasive aspergillosis: review of 2,121 published cases. Rev Infect Dis. 1990 Nov-Dec;12(6):1147-201.

3. Turner JH, Soudry E, Nayak JV, Hwang PH. Survival outcomes in acute invasive fungal sinusitis: a systematic review and quantitative synthesis of published evidence. Laryngoscope. 2013;123(5):1112-8.

4. Talmi YP, Goldschmied-Reouven A, Bakon $M$, et al. Rhino-orbital and rhino-orbitocerebral mucormycosis. Otolaryngol Head Neck Surg. 2002 Jul;127(1):22-31.

5. Kennedy CA, Adams GL, Neglia JP, Giebink GS. Impact of surgical treatment on paranasal fungal infections in bone marrow transplant patients. Otolaryngol Head Neck Surg. 1997 Jun;1 16(6 Pt 1):610-6.

6. Gillespie MB, O'Malley BW, Jr., Francis HW An approach to fulminant invasive fungal rhinosinusitis in the immunocompromised host. Arch Otolaryngol Head Neck Surg 1998 May; 124(5):520-6.

7. Suslu AE, Ogretmenoglu O, Suslu N, Yucel OT, Onerci TM. Acute invasive fungal rhinosinusitis: our experience with 19 patients. Eur Arch Otorhinolaryngol. 2009 Jan;266(1):7782

8. Gamba JL, Woodruff WW, Djang WT, Yeates AE. Craniofacial mucormycosis: assessment with CT. Radiology. 1986;160(1):207-12.

9. Aribandi M, McCoy VA, Bazan C, 3rd Imaging features of invasive and noninvasive fungal sinusitis: a review. Radiographics. 2007 Sep-Oct;27(5):1283-96.

10. DelGaudio JM, Swain RE, Jr., Kingdom TT, Muller S, Hudgins PA. Computed tomographic findings in patients with invasive fungal sinusitis. Arch Otolaryngol Head Neck Surg. 2003 Feb;129(2):236-40.

11. Silverman CS, Mancuso AA. Periantral softtissue infiltration and its relevance to the early detection of invasive fungal sinusitis: CT and MR findings.AJNR Am J Neuroradiol. 1998 Feb;19(2):321-5.

12. Finkelstein A, Contreras D, Pardo J, et al. Paranasal sinuses computed tomography in the initial evaluation of patients with suspected invasive fungal rhinosinusitis. Eur Arch Otorhinolaryngol. 2011 Aug;268(8):1157-1162.

13. Middlebrooks $E H$, Frost $C J$, De Jesus RO Massini TC, Schmalfuss IM, Mancuso AA. Acute Invasive Fungal Rhinosinusitis: A Comprehensive Update of CT Findings and Design of an Effective Diagnostic Imaging Model. AJNR Am J Neuroradiology. 2015;36(8):1529-35.

14. Groppo ER, El-Sayed IH, Aiken AH, Glastonbury CM. Computed tomography and magnetic resonance imaging characteristics of acute invasive fungal sinusitis. Arch Otolaryngol Head Neck Surg. 2011 Oct;137(10):1005-10.

15. Margo CE, Linden C, Strickland-Marmol
LB, Denietolis AL, McCaffrey JC, Kirk N Rhinocerebral mucormycosis with perineural spread. Ophthal Plast Reconstr Surg. 2007 Jul-Aug;23(4):326-7.

16. Orguc S, Yuceturk AV, Demir MA, Goktan C. Rhinocerebral mucormycosis: perineural spread via the trigeminal nerve. J Clin Neurosci. 2005 May;12(4):484-6.

17. Cho HJ, Hong SD, Kim HY, Chung SK, Dhong HJ. Clinical implications of serum galactomannan measurement in patients with acute invasive fungal rhinosinusitis. Rhinology. 2016:54(4):336-41.

18. Shpitzer T, Keller N, Wolf M, et al. Seasonal variations in rhino-cerebral Mucor infection. Ann Otol Rhinol Laryngol. 2005 Sep;114(9):695-8.

Guy Slonimsky, MD

Department of Otolaryngology

Head and Neck Surgery

the Chaim Sheba Medical Center

Tel Hashomer 52621

Israel

Tel: +972-507-210629

Fax: +972-3-5305387

E-mail guyslow@gmail.com 